\title{
On the spectrum of a Robin Laplacian in a planar waveguide
}

\author{
Alex F. Rossini
}

\begin{abstract}
We consider the Laplace operator in a planar waveguide, i.e., an infinite two-dimensional straight strip of constant width, with particular types of Robin boundary conditions. We study the essential spectrum of the corresponding Laplacian when the boundary coupling function has a limit at infinity. Furthermore, we derive sufficient conditions for the existence of discrete spectrum.
\end{abstract}

MSC (2010): 47F05 (primary); 47B25, 81Q05 (secondary).

Running-head title: Robin planar waveguide.

Keywords: planar waveguides, discrete spectrum, Robin boundary conditions.

\section{Introduction}

There are different ways of confining a quantum particle in long and thin structures, the socalled quantum waveguides in suitable subsets $\Omega_{\epsilon}$ of the space $\mathbb{R}^{3}$ or the plane $\mathbb{R}^{2}, 6,14,13,15$, 7. 19. A usual possibility, in two dimensions, is to model the waveguide by a curved strip of constant width which is squeezed between two curves; in this region one considers the Laplacian subject to Dirichlet [6, 8, 14, Neumann [11, 7] or Robin boundary conditions [5, 12, 16].

Our main interest in this paper is to describe the precise location of the essential spectrum of a Robin Laplacian $-\Delta_{\alpha}^{\Omega_{\epsilon}}$, and study the existence of eigenvalues below the essential spectrum, in a straight quantum waveguide $\Omega_{\epsilon}$; see [3, 12, 16, 20, for related references.

The description of the model here studied is as follows. Given a positive number $\epsilon$, consider the infinite straight strip $\Omega_{\epsilon}=\mathbb{R} \times I$, where $I=(0, \epsilon)$ is a bounded interval. The operator $-\Delta_{\alpha}^{\Omega_{\epsilon}}$ acts as the Laplacian in the Hilbert space $\mathrm{L}^{2}\left(\Omega_{\epsilon}\right)$ with certain Robin conditions at the boundary $\partial \Omega_{\epsilon}$. More specifically, given a bounded real-valued function $\alpha(x)$ on $\mathbb{R}$, the classical version of such conditions are

$$
\left\{\begin{array}{c}
-\frac{\partial \psi}{\partial y}(x, 0)-\alpha(x) \psi(x, 0)=0 \\
\frac{\partial \psi}{\partial y}(x, \epsilon)+\alpha(x) \psi(x, \epsilon)=0
\end{array},\right.
$$

for each $x \in \mathbb{R}$ and each $\psi \in \operatorname{dom}\left(-\Delta_{\alpha}^{\Omega_{\epsilon}}\right)$. A related type of boundary conditions has been considered in [16]; there the author has investigated spectral properties of the Laplacian by 
imposing (usual) Robin conditions, i.e., without changing the sign of the Robin parameter $\alpha$ as in (1), whose classical version are

$$
\left\{\begin{array}{c}
-\frac{\partial \psi}{\partial y}(x, 0)+\alpha(x) \psi(x, 0)=0 \\
\frac{\partial \psi}{\partial y}(x, \epsilon)+\alpha(x) \psi(x, \epsilon)=0
\end{array},\right.
$$

where $\alpha(x)$ is positive for all $x \in \mathbb{R}$. Considering this case and under the hypothesis that $\alpha$ tends to a constant at infinity, the essential spectrum of the Laplacian was determined and a sufficient condition for the existence of discrete spectrum was given. The strategy in [16 to prove the existence of at least one isolated eigenvalue, below the threshold of the essential spectrum, was a variational one based on [8], and the method of Neumann Bracketing was employed to find the location of the essential spectrum.

It is a question here whether there are any similar results when one chooses our boundary conditions (1). This change of sign of the Robin parameter leads to nonpositive quadratic forms and, in this context, we were able to get similar results as in [16]. Note that here the location of the essential spectrum was obtained by means the an idea in [2].

Let $-\Delta_{\alpha}^{\Omega_{\epsilon}}$ denote the Laplacian with $\operatorname{dom}\left(-\Delta_{\alpha}^{\Omega_{\epsilon}}\right)=\left\{\psi \in H^{2}\left(\Omega_{\epsilon}\right) ; \psi\right.$ satisfies (1) $\}$. Note that $-\alpha_{0}^{2}$ is the first eigenvalue of the Laplacian $-\Delta_{\alpha_{0}}^{I}$ in $\mathrm{L}^{2}(I)$ with $\psi(x) \in \operatorname{dom}\left(-\Delta_{\alpha_{0}}^{I}\right)$ if $\phi \in H^{2}(I)$ and satisfying

$$
\left\{\begin{array}{c}
-\psi^{\prime}(0)-\alpha_{0} \psi(0)=0 \\
\psi^{\prime}(\epsilon)+\alpha_{0} \psi(\epsilon)=0
\end{array} .\right.
$$

We are going to show that, under some conditions as $\lim _{|x| \rightarrow+\infty}\left(\alpha-\alpha_{0}\right)=0$,

$$
\sigma_{\mathrm{ess}}\left(-\Delta_{\alpha}^{\Omega_{\epsilon}}\right)=\sigma_{\mathrm{ess}}\left(-\Delta_{\alpha_{0}}^{\Omega_{\epsilon}}\right)=\left[-\alpha_{0}^{2},+\infty\right)
$$

and

$$
\sigma\left(-\Delta_{\alpha}^{\Omega_{\epsilon}}\right) \cap\left(-\infty,-\alpha_{0}^{2}\right) \neq \emptyset .
$$

The operators are introduced as the unique self-adjoint operators associated with appropriate quadratic forms and the boundary conditions should be understood in the sense of traces (see more details in Sections 2 and 3).

The paper is organized as follow. In Section 2 we introduce a Robin Laplacian in a bounded interval (transversal section), show that its essential spectrum is empty and explicitly compute its eigenvalues. The change of sign of the Robin parameter $\alpha$ leads to a negative first eigenvalue (and equal to $-\alpha^{2}$ ), whereas all the others are positive. In Section 3 we pass to the corresponding study in an infinite straight and narrow strip. We show, via quadratic forms, that the operator $-\Delta_{\alpha}^{\Omega_{\epsilon}}$ is self-adjoint (Theorem [3.6). Finally, in Section 4 , we find the essential spectrum of such Robin Laplacian operator, and gives sufficient conditions for the existence of discrete spectrum.

\section{Transversal Robin Laplacian}

Initially some results will be presented to our Robin Laplacian in the interval (transversal section) $I=(0, \epsilon)$; they will be important ahead. Here, we find that the Laplacian operator $-\Delta_{\alpha}^{I}$ (classic) in $\mathrm{L}^{2}(I)$ is self-adjoint by using the theory of quadratic forms. 
Consider the operator

$$
-\Delta_{\alpha}^{I}: \operatorname{dom}\left(-\Delta_{\alpha}^{I}\right) \rightarrow \mathrm{L}^{2}(I)
$$

with $\operatorname{dom}\left(-\Delta_{\alpha}^{I}\right)=\left\{\psi \in H^{2}(I) ; \psi\right.$ satisfies (4) $\}$,

$$
\left\{\begin{array}{c}
-\psi^{\prime}(0)-\alpha \psi(0)=0 \\
\psi^{\prime}(\epsilon)+\alpha \psi(\epsilon)=0
\end{array} .\right.
$$

Let $b_{\alpha} \geq-|\alpha|^{2}$ be the closed sesquilinear form $\mathcal{H}$, with domain dom $b_{\alpha}=H^{1}(I) \sqsubseteq \mathrm{L}^{2}(I)$,

$$
b_{\alpha}(\phi, \psi)=\int_{0}^{\epsilon} \overline{\phi^{\prime}(y)} \psi^{\prime}(y) \mathrm{d} y+\alpha(\overline{\phi(\epsilon)} \psi(\epsilon)-\overline{\phi(0)} \psi(0)) .
$$

Theorem 2.1 Let $\alpha \in \mathbb{R}-\{0\}$. Then, the (negative) Laplacian operator $-\Delta_{\alpha}^{I}$ is the unique self-adjoint operator associated with the sesquilinear form $b_{\alpha}$, that is,

$$
b_{\alpha}(\phi, \psi)=\left(\phi,-\Delta_{\alpha}^{I} \psi\right)
$$

for each $\phi \in \operatorname{dom} b_{\alpha}$, and $\psi \in \operatorname{dom}\left(-\Delta_{\alpha}^{I}\right)$.

Proof. We will first prove that $b_{\alpha}$ is closed and lower bounded. Denote by \|\| the norm in $\mathrm{L}^{2}(I)$; we have that

$$
b_{\alpha}(\phi) \geq-|\alpha|^{2}\|\phi\|^{2}, \quad \forall \phi \in \operatorname{dom} b_{\alpha} .
$$

Indeed, first note that for all $a>0$ we have $2\left\|\phi^{\prime}\right\|\|\phi\| \leq a^{2}\|\phi\|^{2}+\frac{1}{a^{2}}\left\|\phi^{\prime}\right\|^{2}$. If $\phi \in \operatorname{dom} b_{\alpha}$, then

$$
\begin{aligned}
\left(|\phi(1)|^{2}-|\phi(0)|^{2}\right) & =\int_{0}^{1} \frac{\mathrm{d}}{\mathrm{d} x}|\phi|^{2} \mathrm{~d} x=\int_{0}^{1}\left(\overline{\phi^{\prime}} \phi+\phi^{\prime} \bar{\phi}\right) \mathrm{d} x \\
& \leq 2\left\|\phi^{\prime}\right\|\|\phi\| \leq a^{2}\|\phi\|^{2}+\frac{1}{a^{2}}\left\|\phi^{\prime}\right\|^{2} .
\end{aligned}
$$

Consequently,

$$
b_{\alpha}(\phi) \geq\left(1-\frac{|\alpha|}{a^{2}}\right)\left\|\phi^{\prime}\right\|^{2}-|\alpha| a^{2}\|\phi\|^{2} .
$$

Choosing $a=\sqrt{|\alpha|}$, we obtain $b_{\alpha}(\phi) \geq-|\alpha|^{2}\|\phi\|^{2}$. Note that by choosing $a=\sqrt{2|\alpha|}$, we will have

$$
b_{\alpha}(\phi) \geq \frac{1}{2}\left\|\phi^{\prime}\right\|^{2}-2|\alpha|^{2}\|\phi\|^{2} .
$$

By inequality (5) and since $H^{1}(I) \subset \mathrm{C}[0, \epsilon]$, with continuous injection, it follows that $b_{\alpha}$ is closed.

Denote by $T_{b_{\alpha}}$ the unique self-adjoint operator associated with the form $b_{\alpha}$, then $T_{b_{\alpha}}=-\Delta_{\alpha}^{I}$ with $\operatorname{dom} T_{b_{\alpha}}=\operatorname{dom}\left(-\Delta_{\alpha}^{I}\right)$. Indeed, if $\psi \in \operatorname{dom}\left(-\Delta_{\alpha}^{I}\right)$ then, by integration by parts, we have the equality $b_{\alpha}(\phi, \psi)=\left(\phi,-\psi^{\prime \prime}\right)_{L^{2}(I)}$; whence we obtain $\left.T_{b_{\alpha}}\right|_{\operatorname{dom}\left(-\Delta_{\alpha}^{I}\right)}=-\Delta_{\alpha}^{I}$, because $\operatorname{dom}\left(-\Delta_{\alpha}^{I}\right) \subseteq \operatorname{dom} T_{b_{\alpha}}$, and so $T_{b_{\alpha}}=-\Delta_{\alpha}^{I}$.

Let $\psi \in \operatorname{dom} T_{b_{\alpha}}$ and $\eta:=T_{b_{\alpha}} \psi \in \mathrm{L}^{2}(I)$ then $(\phi, \eta)_{\mathrm{L}^{2}(I)}=b_{\alpha}(\phi, \psi)$, i.e,

$$
\int_{0}^{\epsilon} \bar{\phi} \eta \mathrm{d} x=\int_{0}^{\epsilon} \bar{\phi}^{\prime} \psi^{\prime} \mathrm{d} x+\alpha(\bar{\phi}(\epsilon) \psi(\epsilon)-\bar{\phi}(0) \psi(0)) .
$$


Following the ideas in [17, Example VI. 2.16, let $z \in H^{1}(I)$ a primitive of $\eta$, i.e., $z^{\prime}=\eta$, then

$$
\int_{0}^{\epsilon} \bar{\phi} \eta \mathrm{d} x=\int_{0}^{\epsilon} \bar{\phi} z^{\prime} \mathrm{d} x=-\int_{0}^{\epsilon} \bar{\phi}^{\prime} z \mathrm{~d} x+(\bar{\phi}(\epsilon) z(\epsilon)-\bar{\phi}(0) z(0)) .
$$

Comparing (6) and (7) the equality

$$
\int_{0}^{\epsilon} \bar{\phi}^{\prime}\left(\psi^{\prime}+z\right) \mathrm{d} x+\bar{\phi}(\epsilon)[\alpha \psi(\epsilon)-z(\epsilon)]+\bar{\phi}(0)[-\alpha \psi(0)+z(0)]=0, \forall \phi \in H^{1}(I),
$$

holds true. In particular, if $\phi \in \mathrm{C}_{0}^{\infty}(0, \epsilon)$ then $\int_{0}^{\epsilon} \bar{\phi}^{\prime}\left(\psi^{\prime}+z\right) \mathrm{d} x=0$, so, $\psi^{\prime}+z=c \quad$ a.e $\quad x \in I$. Therefore, $\psi \in H^{2}(I)$, because $\psi^{\prime}=c-z \in H^{1}(I)$. Moreover, $\psi^{\prime \prime}=-z^{\prime}=-\eta$ and

$$
\left\{\begin{array}{l}
\psi^{\prime}(0)+z(0)=c \\
\psi^{\prime}(\epsilon)+z(\epsilon)=c
\end{array} .\right.
$$

Thus, only remains to verify the Robin condition for $\psi$, and will follow the inclusion $\operatorname{dom} T_{b_{\alpha}} \subseteq \operatorname{dom}\left(-\Delta_{\alpha}^{I}\right)$. Finally, if we replace $\left(\psi^{\prime}+z\right)=c$ in (8) and use integration by parts we deduce

$$
\bar{\phi}(\epsilon)[c+\alpha \psi(\epsilon)-z(\epsilon)]+\bar{\phi}(0)[-c-\alpha \psi(0)+z(0)]=0, \forall \phi \in H^{1}(I) .
$$

Since $\phi$ is arbitrary and in view of (9) the desired conclusion follows.

Remark 2.2 Note that Theorem 2.1 holds for $\alpha=0$, i.e., the Laplacian $-\Delta_{N}^{I}$ with Neumann condition, which is the operator associated with

$$
b_{N}(\phi)=\int_{0}^{\epsilon}\left|\phi^{\prime}\right|^{2} \mathrm{~d} y
$$

$\operatorname{dom} b_{N}=H^{1}(I)$ and $\operatorname{dom}\left(-\Delta_{N}^{I}\right)=\left\{\psi \in H^{2}(I), \psi^{\prime}(0)=\psi^{\prime}(\epsilon)=0\right\}$. In addition, we know that the eigenvalues of $-\Delta_{N}^{I}$ rearranged in an ascending order are given by

$$
\lambda_{0}^{N}=0, \quad \lambda_{n}^{N}=\frac{n^{2} \pi^{2}}{\epsilon^{2}}, \text { with } n \in \mathbb{N} .
$$

The corresponding normalized eigenfunctions are:

$$
\psi_{n}^{N}(y)=\left\{\begin{array}{ll}
\sqrt{\frac{1}{\epsilon}}, & \text { if } n=0 \\
\sqrt{\frac{2}{\epsilon}} \cos \left(\frac{n \pi y}{\epsilon}\right), & \text { if } n \geq 1
\end{array} .\right.
$$

The sequence $\left\{\psi_{n}^{N}\right\}_{n=1}^{\infty}$ is a orthonormal basis of $\mathrm{L}^{2}(I)$ and since $\lambda_{n}^{N} \rightarrow \infty$ when $n \rightarrow \infty$, we have that the essential spectrum $\sigma_{\mathrm{ess}}\left(-\Delta_{N}^{I}\right)$ of operator $-\Delta_{N}^{I}$ is empty. Therefore, the spectrum $\sigma\left(-\Delta_{N}^{I}\right)$ of operator $-\Delta_{N}^{I}$ is equal to discrete spectrum $\sigma_{\mathrm{disc}}\left(-\Delta_{N}^{I}\right)$, that is,

$$
\sigma\left(-\Delta_{N}^{I}\right)=\sigma_{\text {disc }}\left(-\Delta_{N}^{I}\right)=\left\{\lambda_{n}^{N}\right\}_{n=0}^{\infty} .
$$

For future reference, let $\psi_{n}^{D}(x)=\sqrt{\frac{2}{\epsilon}} \sin ((n \pi y) / \epsilon), n \geq 1$, the normalized eigenfunctions associated with the Dirichlet Laplacian $-\Delta_{D}^{I}$, i.e., the operator associated with the quadratic form

$$
b_{D}(\phi)=\int_{0}^{\epsilon}\left|\phi^{\prime}\right|^{2} \mathrm{~d} x
$$

$\operatorname{dom} b_{D}=H_{0}^{1}(I)$ and $\operatorname{dom}\left(-\Delta_{D}^{I}\right)=\left\{\psi \in W^{2,2}(I), \psi(0)=\psi(\epsilon)=0\right\}$. See [19] for more details. 


\subsection{Absence of Essential spectrum}

Here we determine the essential spectrum of our transveral Robin Laplacian operator $\Delta_{\alpha}^{I}$. Inspired by [18, see section (6.2), we will conclude that $\sigma_{\mathrm{ess}}\left(-\Delta_{\alpha}^{I}\right)=\emptyset$. To prove this fact, we need the following result (Lemma 2.3 ahead) from [17] Theorem V.2.20.

Lemma 2.3 Let $\left\{\psi_{n}^{N}\right\}_{n=0}^{\infty}$ be a complete orthonormal family in $\mathcal{H}$ (Hilbert space) and let $\left\{\psi_{n}\right\}_{n=0}^{\infty}$ be a sequence such that $\sum_{n=0}^{\infty}\left\|\psi_{n}-\psi_{n}^{N}\right\|_{2}^{2}<\infty$. Then, $\psi_{n}$ is a basis of $\mathcal{H}$, and so, if $0=\sum_{n=0}^{\infty} c_{n}^{\psi} \psi_{n}$, then all $c_{n}^{\psi}=0$.

We will see in the next lemma, that the elements

$$
\psi_{0}(y)=c e^{-\alpha y}, \quad \psi_{n}(y)=\frac{n \pi}{\left(n^{2} \pi^{2}+\alpha^{2} \epsilon^{2}\right)^{1 / 2}}\left(\psi_{n}^{N}-\frac{\alpha \epsilon}{n \pi} \psi_{n}^{D}\right), n \geq 1
$$

consist of an orthonormal basis of $\mathrm{L}^{2}(I)$. Moreover, in Subsection 2.2. theses elements are shwon to be eigenvectors of $-\Delta_{\alpha}^{I}$, associated with the eigenvalues, respectively,

$$
\lambda_{0}=-\alpha^{2} \quad \text { and } \quad \lambda_{n}=\left(n^{2} \pi^{2}\right) / \epsilon^{2}, n \geq 1 .
$$

Lemma 2.4 The sequence $\left\{\psi_{n}\right\}_{n=0}^{\infty}$ is a complete orthonormal family in $\mathrm{L}^{2}(I)$.

Proof. Indeed, given $\phi \in \mathrm{L}^{2}(I)$ and $\psi=\sum_{n=0}^{\infty} c_{n}^{\psi} \psi_{n}$ it follows that

$$
(\phi, \psi)_{\mathrm{L}^{2}(I)}=\lim _{m \rightarrow \infty}\left(\phi, \sum_{n=0}^{m} c_{n}^{\psi} \psi_{n}\right)_{\mathrm{L}^{2}(I)}
$$

For $\psi=0$ and $\phi=\psi_{k}$, it follows by (12) and orthogonality that $c_{k}^{\psi}=0$, for each $k=0,1, \ldots$ On the other hand, we have

$$
\sum_{n=0}^{\infty}\left\|\psi_{n}-\psi_{n}^{N}\right\|_{\mathrm{L}^{2}(I)}^{2}<\infty .
$$

Indeed, for $n \geq 1$, we have

$$
\left\|\psi_{n}-\psi_{n}^{N}\right\|_{\mathrm{L}^{2}(I)}^{2}=2-\frac{2 n \pi}{\sqrt{n^{2} \pi^{2}+\alpha^{2} \epsilon^{2}}} \rightarrow 0, \quad n \rightarrow \infty .
$$

Since the function $f(x)=2-\frac{2 x \pi}{\sqrt{x^{2} \pi^{2}+\alpha^{2} \epsilon^{2}}}$ is decreasing and $\int_{1}^{\infty} f(x) \mathrm{d} x<\infty$, the integral test for convergence give us that $\Sigma_{n=1}^{\infty} f(n)<+\infty$, consequently, $\sum_{n=0}^{\infty}\left\|\psi_{n}-\psi_{n}^{N}\right\|_{\mathrm{L}^{2}(I)}^{2}$ is convergent. Therefore, by Lemma 2.3, the desired result follows.

Theorem 2.5 Let us suppose $\alpha \in \mathbb{R}-\{0\}$, then the transversal Robin Laplacian $-\Delta_{\alpha}^{I}$ has purely discrete spectrum, i.e., the essential spectrum $\sigma_{\mathrm{ess}}\left(-\Delta_{\alpha}^{I}\right)$ of operator $-\Delta_{\alpha}^{I}$ is empty.

Proof. By Theorem 11.3.13 in 4, it follows that $\sigma_{\mathrm{ess}}\left(-\Delta_{\alpha}^{I}\right)=\emptyset$, since the sequence $\left\{\psi_{n}\right\}_{n=0}^{\infty}$ is a orthonormal basis of $\mathrm{L}^{2}(I)$ and $\lambda_{n} \rightarrow \infty$ when $n \rightarrow \infty$. Therefore,

$$
\sigma\left(-\Delta_{\alpha}^{I}\right)=\sigma_{\text {disc }}\left(-\Delta_{\alpha}^{I}\right)=\left\{\lambda_{n}\right\}_{n=0}^{\infty} .
$$




\subsection{Point spectrum of the transversal Laplacian}

To obtain the point spectrum of $-\Delta_{\alpha}^{I}$, let us to determine $\lambda \in \mathbb{R}$ for which there exists $0 \neq \psi \in H^{2}(I)$, normalized in $\mathrm{L}^{2}(I)$, satisfying

$$
-\psi^{\prime \prime}=\lambda \psi
$$

and the boundary conditions $(\alpha \neq 0)$,

$$
\left\{\begin{array}{r}
-\psi^{\prime}(0)-\alpha \psi(0)=0 \\
\psi^{\prime}(\epsilon)+\alpha \psi(\epsilon)=0
\end{array} .\right.
$$

If $\lambda>0$ we know that the general solution (classic) of (13) is given by

$$
\psi(x)=A \sin (\sqrt{\lambda} x)+B \cos (\sqrt{\lambda} x)
$$

with $A, B \in \mathbb{C}$ determined by the Robin condition and the normalization condition. Thus, by imposing the Robin conditions on the general solution we obtain the following system

$$
\left[\begin{array}{cc}
\sqrt{\lambda} & \alpha \\
\sqrt{\lambda} \cos (\sqrt{\lambda} \epsilon)+\alpha \sin (\sqrt{\lambda} \epsilon) & \alpha \cos (\sqrt{\lambda} \epsilon)-\lambda \sin (\sqrt{\lambda} \epsilon)
\end{array}\right]\left[\begin{array}{c}
A \\
B
\end{array}\right]=0 .
$$

Since we are interested in nonzero solution, we must impose that the determinant of the above matrix is zero. This requirement enables us to obtain $\lambda$ explicitly:

$$
\left(\alpha^{2}+\lambda\right) \sin (\sqrt{\lambda} \epsilon)=0
$$

i.e.,

$$
\lambda=-\alpha^{2} \quad \text { or } \quad \lambda=\frac{n^{2} \pi^{2}}{\epsilon^{2}}, n \in \mathbb{Z} .
$$

Since the system (16) is equivalent to the equation $\sqrt{\lambda} A+\alpha B=0$, we can express $A$ in function of $B$, i.e, $A=-\frac{\alpha}{\sqrt{\lambda}} B$. Therefore, the corresponding eigenfunction to $\lambda=\frac{n^{2} \pi^{2}}{\epsilon^{2}}, n \geq 1$, is given by

$$
\psi_{n}(x)=B\left(-\frac{\alpha \epsilon}{n \pi} \sin ((n \pi x) / \epsilon)+\cos ((n \pi x) / \epsilon)\right),
$$

with $1=|B|^{2} \epsilon\left[\frac{1}{2}\left(1+\frac{\alpha^{2} \epsilon^{2}}{n^{2} \pi^{2}}\right)\right]$.

Next, for $n \geq 1$ and choosing $B>0$ we have

$$
\psi_{n}(x)=\frac{n \pi}{\left(n^{2} \pi^{2}+\alpha^{2} \epsilon^{2}\right)^{1 / 2}}\left(\sqrt{\frac{2}{\epsilon}} \cos ((n \pi x) / \epsilon)-\frac{\alpha \epsilon}{n \pi} \sqrt{\frac{2}{\epsilon}} \sin ((n \pi x) / \epsilon)\right),
$$

one still has the following,

$$
\psi_{n}(x)=\frac{n \pi}{\left(n^{2} \pi^{2}+\alpha^{2} \epsilon^{2}\right)^{1 / 2}}\left(\psi_{n}^{N}-\frac{\alpha \epsilon}{n \pi} \psi_{n}^{D}\right)
$$

with $\psi_{n}^{D}:=\sqrt{\frac{2}{\epsilon}} \sin ((n \pi x) / \epsilon), n \geq 1$. Recall that $\psi_{n}^{D}$ are the eigenfunctions of $-\Delta_{D}^{I}$, the Laplacian operator with Dirichlet boundary condition at the interval $I$.

Suppose that $\lambda<0$, then the general solution is of the form

$$
\psi(x)=A e^{\sqrt{\mu} x}+B e^{-\sqrt{\mu} x},
$$


with $\mu=-\lambda$. Imposing the boundary conditions we obtain the system

$$
\left[\begin{array}{cc}
\sqrt{\mu}+\alpha & \alpha-\sqrt{\mu} \\
\sqrt{\mu} e^{\sqrt{\mu} \epsilon}+\alpha e^{\sqrt{\mu} \epsilon} & \alpha e^{-\sqrt{\mu} \epsilon}-\sqrt{\mu} e^{-\sqrt{\mu} \epsilon}
\end{array}\right]\left[\begin{array}{l}
A \\
B
\end{array}\right]=0 .
$$

Again, assuming that the determinant is null, follows the equality:

$$
\left(\alpha^{2}-\mu\right)\left(e^{-\sqrt{\mu} \epsilon}-e^{\sqrt{\mu} \epsilon}\right)=0
$$

since $\mu \neq 0$, we have $\mu=\alpha^{2}$, i.e., $\lambda=-\alpha^{2}$. It follows from $\psi^{\prime}(0)+\alpha \psi(0)=0$ that the eigenfunction associated with the negative eigenvalue $\lambda=-\alpha^{2}$ is

$$
\psi(x)=c e^{-\alpha x}, \quad \text { with } \quad c^{-1}=\left\|e^{-\alpha x}\right\|_{\mathrm{L}^{2}(I)} .
$$

If $\lambda=0$ then the general solution is $\psi(x)=A x+B$ and from the boundary conditions it follows that $A=B=0$. Therefore, $\lambda=0$ is not an eigenvalue.

In short, $\lambda_{0}:=-\alpha^{2}$ is the first eigenvalues (negative) of the Robin Laplacian on $I$ ( recall the self-adjointness of $\left.-\Delta_{\alpha}^{I}\right)$ associated with the normalized eigenfunction $\phi_{0}(y)=c e^{-\alpha y}, c>0$, i.e.,

$$
-\phi_{0}^{\prime \prime}=\lambda_{0} \phi_{0}, \quad 0<\phi_{0} \in H^{2}(I) \quad \text { and } \quad \int_{0}^{\epsilon}\left|\phi_{0}\right|^{2} \mathrm{~d} y=1,
$$

moreover, it satisfies the Robin boundary conditions (14).

\section{$3 \quad$ Infinite and straight planar strips}

The purpose of this section is to found that the classic Laplacian $-\Delta_{\alpha}^{\Omega_{\epsilon}}$ in $\mathrm{L}^{2}\left(\Omega_{\epsilon}\right)$, with a suitable domain, is self-adjoint. For this purpose a convenient sesquilinear form $b_{\alpha}^{\Omega_{\epsilon}}$ has been introduced, whose definition will be made precise below.

Under certain conditions on $\alpha$ at infinity, it is possible to prove the existence of isolated bound states, i.e., the existence of eigenvalues (of finite multiplicity) below the essential spectrum $\sigma_{\text {ess }}\left(-\Delta_{\alpha}^{\Omega_{\epsilon}}\right)$ of Laplacian. For this purpose, we follow some ideas in [2, 15, 16].

The closed sesquilinear form of interest is $b_{\alpha}^{\Omega_{\epsilon}} \geq-\|\alpha\|_{\infty}^{2}$ in $\mathrm{L}^{2}\left(\Omega_{\epsilon}\right)$, with domain the Hilbert space $H^{1}\left(\Omega_{\epsilon}\right)$,

$$
\begin{aligned}
b_{\alpha}^{\Omega_{\epsilon}}(\phi, \psi) & =\int_{\Omega_{\epsilon}} \overline{\nabla \phi}(x, y) \nabla \psi(x, y) \mathrm{d} x \mathrm{~d} y \\
& +\int_{\mathbb{R}} \alpha(x)(\overline{\operatorname{tr}(\phi)}(x, \epsilon) \operatorname{tr}(\psi)(x, \epsilon)-\overline{\operatorname{tr}(\phi)}(x, 0) \operatorname{tr}(\psi)(x, 0)) \mathrm{d} x,
\end{aligned}
$$

where $\operatorname{tr}(\phi)$ denotes the trace in $\mathrm{L}^{2}\left(\partial \Omega_{\epsilon}\right)$ of $\phi \in H^{1}\left(\Omega_{\epsilon}\right)$. Consider the negative Laplacian

$$
-\Delta_{\alpha}^{\Omega_{\epsilon}}: \operatorname{dom}\left(-\Delta_{\alpha}^{\Omega_{\epsilon}}\right) \rightarrow \mathrm{L}^{2}\left(\Omega_{\epsilon}\right)
$$

with $\operatorname{dom}\left(-\Delta_{\alpha}^{\Omega_{\epsilon}}\right)=\left\{\psi \in H^{2}\left(\Omega_{\epsilon}\right) ; \psi\right.$ satisfies (18) $\}$,

$$
\left\{\begin{array}{c}
-\frac{\partial \psi}{\partial y}(x, 0)-\alpha(x) \psi(x, 0)=0 \\
\frac{\partial \psi}{\partial y}(x, \epsilon)+\alpha(x) \psi(x, \epsilon)=0
\end{array} ;\right.
$$


in this section we require $\alpha(x) \in W^{1, \infty}(\mathbb{R})$.

A lower bound for $b_{\alpha}^{\Omega_{\epsilon}}$ is initially obtained for $\left.\phi\right|_{\Omega_{\epsilon}}$ with $\phi \in \mathrm{C}_{0}^{\infty}\left(\mathbb{R}^{2}\right)$ and, by density, for each $\phi \in H^{1}\left(\Omega_{\epsilon}\right)$. Note that $\operatorname{tr}\left(\left.\phi\right|_{\Omega_{\epsilon}}\right)=\left.\phi\right|_{\Omega_{\epsilon}}$ in $L^{2}\left(\partial \Omega_{\epsilon}\right)$, consequently

$$
b_{\alpha}^{\Omega_{\epsilon}}(\phi) \geq \int_{\mathbb{R}}\left[\int_{0}^{\epsilon}\left|\frac{\partial \phi}{\partial y}\right|^{2} \mathrm{~d} y+\alpha(x)\left(|\phi(x, \epsilon)|^{2}-|\phi(x, 0)|^{2}\right)\right] \mathrm{d} x,
$$

since $\phi(x, \cdot) \in H^{1}(0, \epsilon)$ for a.e. $x \in \mathbb{R}$. By an argument in Theorem 2.1, we obtain

$$
b_{\alpha}^{\Omega_{\epsilon}}(\phi) \geq-\|\alpha\|_{\infty}^{2} \int_{\Omega_{\epsilon}}|\phi|^{2} \mathrm{~d} x \mathrm{~d} y
$$

for each $\left.\phi\right|_{\Omega_{\epsilon}}$ with $\phi \in \mathrm{C}_{0}^{\infty}\left(\mathbb{R}^{2}\right)$. Let $\phi \in H^{1}\left(\Omega_{\epsilon}\right)$ and $\left\{\phi_{m}\right\}_{m=1}^{\infty}$ a sequence in $\mathrm{C}_{0}^{\infty}\left(\mathbb{R}^{2}\right)$ such that $\left.\phi_{m}\right|_{\Omega_{\epsilon}} \rightarrow \phi$ in $H^{1}\left(\Omega_{\epsilon}\right)$. Then, for each $\phi \in H^{1}\left(\Omega_{\epsilon}\right)$ one finds that

$$
b_{\alpha}^{\Omega_{\epsilon}}(\phi) \geq-\beta\|\phi\|^{2}, \quad \text { with } \quad \beta=\|\alpha\|_{\infty}^{2} .
$$

By standard arguments we can verify that $b_{\alpha}^{\Omega_{\epsilon}}$ is closed.

In the next theorem we show the self-adjointness of $-\Delta_{\alpha}^{\Omega_{\epsilon}}$, i.e., that $-\Delta_{\alpha}^{\Omega_{\epsilon}}$ is the Rodin Laplacian, what we mean as the operator associated with the form $b_{\alpha}^{\Omega_{\epsilon}}$.

Theorem 3.6 Suppose that $\alpha \in W^{1, \infty}(\mathbb{R})$. Then, the negative Laplacian $-\Delta_{\alpha}^{\Omega_{\epsilon}}$ is the (unique) self-adjoint operator associated with the sesquilinear form $b_{\alpha}^{\Omega_{\epsilon}}$, i.e.,

$$
b_{\alpha}^{\Omega_{\epsilon}}(\phi, \psi)=\left(\phi,-\Delta_{\alpha}^{\Omega_{\epsilon}} \psi\right)_{\mathrm{L}^{2}}
$$

for $\phi \in \operatorname{dom} b_{\alpha}^{\Omega_{\epsilon}}, \psi \in \operatorname{dom}\left(-\Delta_{\alpha}^{\Omega_{\epsilon}}\right)$.

The proof is presented through Lemmas 3.7 and 3.8 The first lemma gives some information on the domain of $T_{b_{\alpha}^{\Omega_{\epsilon}}}$, associated with $b_{\alpha}^{\Omega_{\epsilon}}$. It shows that $\operatorname{dom} T_{b_{\alpha}^{\Omega_{\epsilon}}} \subset \operatorname{dom}\left(-\Delta_{\alpha}^{\Omega_{\epsilon}}\right)$. The second one concludes that $T_{b_{\alpha}^{\Omega_{\epsilon}}}$ is an extension of $-\Delta_{\alpha}^{\Omega_{\epsilon}}$. Therefore, we obtain the equality $T_{b_{\alpha}^{\Omega_{\epsilon}}}=$ $-\Delta_{\alpha}^{\Omega_{\epsilon}}$.

Lemma 3.7 Suppose $\alpha \in W^{1, \infty}(\mathbb{R})$. For each $F \in \mathrm{L}^{2}\left(\Omega_{\epsilon}\right)$, every solution $\psi \in H^{1}\left(\Omega_{\epsilon}\right)$ of problem

$$
b_{\alpha}^{\Omega_{\epsilon}}(\phi, \psi)=(\phi, F)_{\mathrm{L}^{2}\left(\Omega_{\epsilon}\right)}, \forall \phi \in \operatorname{dom} b_{\alpha}^{\Omega_{\epsilon}}=H^{1}\left(\Omega_{\epsilon}\right),
$$

belongs to $\operatorname{dom}\left(-\Delta_{\alpha}^{\Omega_{\epsilon}}\right)$. Consequently, $\operatorname{dom} T_{b_{\alpha}^{\Omega_{\epsilon}}} \subset \operatorname{dom}\left(-\Delta_{\alpha}^{\Omega_{\epsilon}}\right)$.

Proof. For $\psi \in H^{1}\left(\Omega_{\epsilon}\right)$, let us introduce the quotient of Newton

$$
\psi_{\delta}(x, y):=\frac{\psi(x+\delta, y)-\psi(x, y)}{\delta}, 0 \neq \delta \in \mathbb{R} .
$$

Since

$$
|\psi(x+\delta, y)-\psi(x, y)|=\left|\int_{0}^{1} \frac{\partial \psi}{\partial x}(x+\delta t, y) \delta \mathrm{d} t\right| \leq|\delta| \int_{0}^{1}\left|\frac{\partial \psi}{\partial x}(x+\delta t, y)\right| \mathrm{d} t,
$$

we have

$$
\int_{\Omega_{\epsilon}}\left|\psi_{\delta}\right|^{2} \mathrm{~d} x \mathrm{~d} y \leq \int_{0}^{1}\left[\int_{\Omega_{\epsilon}}\left|\frac{\partial \psi}{\partial x}(x+\delta t, y)\right|^{2} \mathrm{~d} x \mathrm{~d} y\right] \mathrm{d} t=\int_{\Omega_{\epsilon}}\left|\frac{\partial \psi}{\partial x}(x, y)\right|^{2} \mathrm{~d} x \mathrm{~d} y .
$$


Therefore,

$$
\int_{\Omega_{\epsilon}}\left|\psi_{\delta}\right|^{2} \mathrm{~d} x \mathrm{~d} y \leq\|\psi\|_{1,2}^{2}, \quad \forall 0 \neq \delta \in \mathbb{R} .
$$

If $\psi \in H^{1}\left(\Omega_{\epsilon}\right)$ is a solution to (20), then $\psi_{\delta}$ is a solution to the problem

$$
b_{\alpha}^{\Omega_{\epsilon}}\left(\phi, \psi_{\delta}\right)=\left(\phi, F_{\delta}\right)_{\mathrm{L}^{2}\left(\Omega_{\epsilon}\right)}-\int_{\mathbb{R}} \alpha_{\delta}(x)(\overline{\phi(x, \epsilon)} \psi(x+\delta, \epsilon)-\overline{\phi(x, 0)} \psi(x+\delta, 0)) \mathrm{d} x,
$$

for each $\phi \in H^{1}\left(\Omega_{\epsilon}\right)$. By chosing $\phi=\psi_{\delta}$ and noting that $\left(\phi, F_{\delta}\right)_{\mathrm{L}^{2}\left(\Omega_{\epsilon}\right)}=-\left(\phi_{-\delta}, F\right)_{\mathrm{L}^{2}\left(\Omega_{\epsilon}\right)}$, we obtain that

$$
b_{\alpha}^{\Omega_{\epsilon}}\left(\psi_{\delta}\right)=-\left(\left(\psi_{\delta}\right)_{-\delta}, F\right)_{\mathrm{L}^{2}\left(\Omega_{\epsilon}\right)}-\int_{\mathbb{R}} \alpha_{\delta}(x)\left(\overline{\psi_{\delta}(x, \epsilon)} \psi(x+\delta, \epsilon)-\overline{\psi_{\delta}(x, 0)} \psi(x+\delta, 0)\right) \mathrm{d} x .
$$

For simplicity we write $b_{\alpha}^{\Omega}\left(\psi_{\delta}\right)=b_{1}^{\Omega}\left(\psi_{\delta}\right)+b_{2}^{\Omega}\left(\psi_{\delta}\right)$, with

$$
b_{1}\left(\psi_{\delta}\right)=\int_{\Omega}\left|\nabla \psi_{\delta}\right|^{2} \mathrm{~d} x \mathrm{~d} y \quad \text { and } \quad b_{2}\left(\psi_{\delta}\right)=\int_{\mathbb{R}} \alpha(x)\left(\left|\psi_{\delta}(x, \epsilon)\right|^{2}-\left|\psi_{\delta}(x, 0)\right|^{2}\right) \mathrm{d} x \mathrm{~d} y .
$$

By Schwarz inequality, Cauchy inequality, estimate (21), boundedness of $\alpha$ and $\alpha_{\delta}$, and compact embedding of $H^{1}\left(\Omega_{\epsilon}\right)$ in $\mathrm{L}^{2}\left(\partial \Omega_{\epsilon}\right)$, we can produce the following estimates, for $t>0$,

$$
\begin{gathered}
\left|\left(\left(\psi_{\delta}\right)_{-\delta}, F\right)_{\mathrm{L}^{2}\left(\Omega_{\epsilon}\right)}\right| \leq 2\|F\|_{\mathrm{L}^{2}\left(\Omega_{\epsilon}\right)} \|\left(\left(\psi_{\delta}\right)_{-\delta}\left\|_{\mathrm{L}^{2}\left(\Omega_{\epsilon}\right)} \leq t^{-1}\right\| F\left\|_{\mathrm{L}^{2}\left(\Omega_{\epsilon}\right)}^{2}+t\right\| \psi_{\delta} \|_{1,2}^{2},\right. \\
\left|\int_{\mathbb{R}} \alpha_{\delta}(x)\left(\overline{\psi_{\delta}(x, \epsilon)} \psi(x+\delta, \epsilon)-\overline{\psi_{\delta}(x, 0)} \psi(x+\delta, 0)\right) \mathrm{d} x\right| \\
\leq\|\alpha\|_{\infty}\left\|\psi_{\delta}\right\|_{\mathrm{L}^{2}\left(\partial \Omega_{\epsilon}\right)}\|\psi\|_{\mathrm{L}^{2}\left(\partial \Omega_{\epsilon}\right)} \leq C\left\|\psi_{\delta}\right\|_{1,2}\|\psi\|_{1,2},
\end{gathered}
$$

with $C>0$ independent of $\delta$,

$$
\left|b_{2}^{\Omega}\left(\psi_{\delta}\right)\right| \leq\left.\left|\int_{\Omega} \alpha(x) \frac{\partial}{\partial y}\right| \psi_{\delta}\right|^{2} \mathrm{~d} x \mathrm{~d} y \mid \leq 2\|\alpha\|_{\infty}\left\|\psi_{\delta}\right\|_{\mathrm{L}^{2}\left(\Omega_{\epsilon}\right)}\left\|\partial_{2} \psi_{\delta}\right\|_{\mathrm{L}^{2}\left(\Omega_{\epsilon}\right)} \leq t^{-1}\|\alpha\|_{\infty}^{2}\|\psi\|_{1,2}^{2}+t b_{1}^{\Omega}\left(\psi_{\delta}\right) .
$$

On the one hand, provided that $b_{\alpha}^{\Omega}\left(\psi_{\delta}\right)=b_{1}^{\Omega}\left(\psi_{\delta}\right)+b_{2}^{\Omega}\left(\psi_{\delta}\right)$, one has

$$
b_{\alpha}^{\Omega}\left(\psi_{\delta}\right) \geq(1-t) b_{1}^{\Omega}\left(\psi_{\delta}\right)-t^{-1}\|\alpha\|_{\infty}^{2}\|\psi\|_{1,2}^{2} .
$$

On the other hand, the identity (22) produces

$$
\left|b_{\alpha}^{\Omega}\left(\psi_{\delta}\right)\right| \leq C\left\|\psi_{\delta}\right\|_{1,2}\|\psi\|_{1,2}+\left(t^{-1}\|F\|_{L^{2}\left(\Omega_{\epsilon}\right)}^{2}+t\left\|\psi_{\delta}\right\|_{1,2}^{2}\right) .
$$

So,

$$
(1-t) b_{1}^{\Omega}\left(\psi_{\delta}\right)-t^{-1}\|\alpha\|_{\infty}^{2}\|\psi\|_{1,2}^{2} \leq C\left\|\psi_{\delta}\right\|_{1,2}\|\psi\|_{1,2}+\left(t^{-1}\|F\|_{L^{2}\left(\Omega_{\epsilon}\right)}^{2}+t\left\|\psi_{\delta}\right\|_{1,2}^{2}\right) .
$$

Now, suppose that $0<t<1$ and add $(1-t)\left\|\psi_{\delta}\right\|_{2}^{2}$ to both sides of the above inequality, to obtain

$$
\begin{aligned}
(1-t)\left\|\psi_{\delta}\right\|_{1,2}^{2} & \leq \mathrm{C}\left\|\psi_{\delta}\right\|_{1,2}\|\psi\|_{1,2}+t^{-1}\|F\|_{\mathrm{L}^{2}\left(\Omega_{\epsilon}\right)}^{2}+t\left\|\psi_{\delta}\right\|_{1,2}^{2}+t^{-1}\|\alpha\|_{\infty}^{2}\|\psi\|_{1,2}^{2} \\
& +(1-t)\left\|\psi_{\delta}\right\|_{2}^{2}
\end{aligned}
$$


Therefore,

$0 \leq(2 t-1)\left\|\psi_{\delta}\right\|_{1,2}^{2}+C\left\|\psi_{\delta}\right\|_{1,2}\|\psi\|_{1,2}+\left(t^{-1}\|F\|_{L^{2}\left(\Omega_{\epsilon}\right)}^{2}+t^{-1}\|\alpha\|_{\infty}^{2}\|\psi\|_{1,2}^{2}+(1-t)\|\psi\|_{1,2}^{2}\right)$.

Thus, we assume that $0<t<1 / 2$, so that the dominant term of the quadratic function is negative, consequently we have $\left\|\psi_{\delta}\right\|_{1,2}^{2} \leq \tilde{C}$, with $\tilde{C}$ independent of $\delta$. But, this estimate implies

$$
\sup _{\delta}\left\|\psi_{-\delta}\right\|_{1,2}<\infty
$$

and since $H^{1}\left(\Omega_{\epsilon}\right)$ is reflexive, every bounded sequence has a weakly convergent subsequence, then there is $v \in H^{1}\left(\Omega_{\epsilon}\right)$ and a subsequence $\delta_{k} \rightarrow 0$ such that $\psi_{-\delta_{k}} \stackrel{w}{\rightarrow} v$ in $H^{1}\left(\Omega_{\epsilon}\right)$. Hence,

$$
\begin{aligned}
\int_{\Omega_{\epsilon}} \psi \partial_{x} \phi \mathrm{d} x \mathrm{~d} y & =\int_{\Omega_{\epsilon}} \psi \lim _{\delta_{k} \rightarrow 0} \phi_{\delta_{k}} \mathrm{~d} x \mathrm{~d} y=\lim _{\delta_{k} \rightarrow 0} \int_{\Omega_{\epsilon}} \psi \phi_{\delta_{k}} \mathrm{~d} x \mathrm{~d} y \\
& =-\lim _{\delta_{k} \rightarrow 0} \int_{\Omega_{\epsilon}} \psi_{-\delta_{k}} \phi \mathrm{d} x \mathrm{~d} y=-\int_{\Omega_{\epsilon}} v \phi \mathrm{d} x \mathrm{~d} y .
\end{aligned}
$$

Therefore, $\partial_{x} \psi=v$ in the weak sense, and so $\partial_{x} \psi \in H^{1}\left(\Omega_{\epsilon}\right)$. Consequently, $\partial_{x x} \psi \in \mathrm{L}^{2}\left(\Omega_{\epsilon}\right)$ and $\partial_{y x} \psi \in \mathrm{L}^{2}\left(\Omega_{\epsilon}\right)$. It follows from the standard elliptic regularity theorems (see 9] Theorem 1, page 309) that $\psi \in W_{l o c}^{2,2}\left(\Omega_{\epsilon}\right)$, so $-\Delta \psi=F$ a.e. in $\Omega_{\epsilon}$. Hence, $\partial_{y y} \psi=-\left(F+\partial_{x x} \psi\right) \in \mathrm{L}^{2}\left(\Omega_{\epsilon}\right)$, and therefore $\psi \in W^{2,2}\left(\Omega_{\epsilon}\right)$.

Finally, it remains to verify that $\psi$ satisfies the boundary conditions. After integration by parts

$$
\begin{aligned}
(\phi, F)_{\mathrm{L}^{2}\left(\Omega_{\epsilon}\right)}=b_{\alpha}^{\Omega_{\epsilon}}(\psi, \phi)=(\phi,-\Delta \psi)_{\mathrm{L}^{2}\left(\Omega_{\epsilon}\right)} & +\int_{\mathbb{R}} \overline{\phi(x, 0)}\left[-\partial_{y} \psi(x, 0)-\alpha(x) \psi(x, 0)\right] \mathrm{d} x \\
& +\int_{\mathbb{R}} \overline{\phi(x, \epsilon)}\left[\partial_{y} \psi(x, \epsilon)+\alpha(x) \psi(x, \epsilon)\right] \mathrm{d} x
\end{aligned}
$$

for each $\phi \in H^{1}\left(\Omega_{\epsilon}\right)$. This implies the boundary conditions, because $-\Delta \psi=F$ a.e. in $\Omega_{\epsilon}$ and $\phi$ is arbitrary.

Lemma 3.8 Suppose that $\alpha \in W^{1, \infty}(\mathbb{R})$. Then $T_{\alpha}=-\Delta_{\alpha}^{\Omega_{\epsilon}}$.

Proof. Let $\psi \in \operatorname{dom}\left(-\Delta_{\alpha}^{\Omega_{\epsilon}}\right)$, then $\psi \in W^{2,2}\left(\Omega_{\epsilon}\right)$ and it satisfies the boundary conditions (18). By integration by parts and (18) we obtain, for each $\phi \in \operatorname{dom} b_{\alpha}^{\Omega_{\epsilon}}$, the identity

$$
\begin{aligned}
b_{\alpha}^{\Omega_{\epsilon}}(\phi, \psi)= & \int_{\mathbb{R}} \overline{\phi(x, \epsilon)} \partial_{y} \psi(x, \epsilon) \mathrm{d} x-\int_{\mathbb{R}} \overline{\phi(x, 0)} \partial_{y} \psi(x, 0) \mathrm{d} x-\int_{\Omega} \overline{\phi(x, y)} \Delta \psi(x, y) \mathrm{d} x \mathrm{~d} y \\
& +\int_{\mathbb{R}} \alpha(x) \overline{\phi(x, \epsilon)} \psi(x, \epsilon) \mathrm{d} x-\int_{\mathbb{R}} \alpha(x) \overline{\phi(x, 0)} \psi(x, 0) \mathrm{d} x=(\phi,-\Delta \psi)_{\mathrm{L}^{2}\left(\Omega_{\epsilon}\right)} .
\end{aligned}
$$

Thus, $\psi \in \operatorname{dom} T_{\alpha}$, and it follows that $T_{\alpha}$ is an extension of $-\Delta_{\alpha}^{\Omega_{\epsilon}}$. It follows, by Lemma 3.7 the desired equality. 


\section{The spectrum of the Robin Laplacian in $\Omega_{\epsilon}$}

Here we investigate the spectrum of the operator $-\Delta_{\alpha}^{\Omega_{\epsilon}}$ when the Robin parameter (function) $\alpha$ in $W^{1, \infty}(\mathbb{R})$ satisfies the condition

$$
\lim _{|x| \rightarrow+\infty}\left(\alpha(x)-\alpha_{0}\right)=0,
$$

i.e, given $\delta>0$ there exists $a>0$ such that $\left|\alpha(x)-\alpha_{0}\right|<\delta$ whenever $|x|>a$. Denote $\beta:=\left(\alpha-\alpha_{0}\right)$ and define the functions

$$
\beta_{m}=\left\{\begin{array}{lll}
\alpha-\alpha_{0}, & \text { if } & |x|<m \\
0, & \text { if } & |x| \geq m
\end{array} .\right.
$$

This sequence of bounded functions with compact support converges to $\beta$ in $\mathrm{L}^{\infty}(\mathbb{R})$.

In case that (23) holds, we prove that the essential part $\sigma_{\mathrm{ess}}\left(-\Delta_{\alpha}^{\Omega_{\epsilon}}\right)$ of the spectrum $-\Delta_{\alpha}^{\Omega_{\epsilon}}$ is the interval $\left[-\alpha_{0}^{2}, \infty\right)$. This statement is the content of Theorem 4.10, whose proof is performed in two steps, that is, Propositions 4.11 and and 4.13, whose proofs were inspired in 2. The proof of Proposition 4.11 makes use of the so-called called Weyl criterion for the essential spectrum (see [4, Theorem 11.2.7), which we recall.

Lemma 4.9 (Weyl criterion) Let $T$ be a self-adjoint operator in a complex Hilbert space $\mathcal{H}$. Then, $\lambda \in \sigma_{\text {ess }}(T)$ iff there exists a sequence $\left\{\psi_{n}\right\}_{n=1}^{\infty} \subset \operatorname{dom} T$ such that

1) $\left\|\psi_{n}\right\|=1, \forall n \in \mathbb{N}$;

2) $\psi_{n} \stackrel{w}{\longrightarrow} 0$, as $n \rightarrow \infty$ in $\mathcal{H}$;

3) $(T-\lambda) \psi_{n} \rightarrow 0$, as $n \rightarrow \infty$.

Such a sequence is called a singular Weyl sequence for $T$ at $\lambda$.

Lemma 4.10 For each $\alpha_{0} \in \mathbb{R},\left[-\alpha_{0}^{2},+\infty\right) \subset \sigma_{\text {ess }}\left(-\Delta_{\alpha_{0}}^{\Omega_{\epsilon}}\right)$.

Proof. Let $\lambda \in\left[\mu_{0}, \infty\right)$ with $\mu_{0}=-\alpha_{0}^{2}$. So, one can write $\lambda=\mu_{0}+t$, with $t \in[0,+\infty)$. We denote by $-\Delta^{\mathbb{R}}$ the Laplacian operator in $L^{2}(\mathbb{R})$. It is well known that $\sigma_{\text {ess }}\left(-\Delta^{\mathbb{R}}\right)=[0,+\infty)$. Hence, there is a singular Weyl sequence $\left\{\phi_{n}\right\}_{n=1}^{+\infty}$ for $-\Delta^{\mathbb{R}}$ at $t$. Define the sequence $\left\{\psi_{n}\right\}_{n=1}^{+\infty}$ as $\psi_{n}(x, y)=\phi_{n}(x) \phi_{0}(y)$ with $\phi_{0}=c\left(\alpha_{0}\right) e^{-\alpha_{0} y}$ the eigenfunction (normalized) of $-\Delta_{\alpha_{0}}^{I}$, associated with the first eigenvalue $\mu_{0}=-\alpha_{0}^{2}$. Note that $\left\{\psi_{n}\right\}_{n=1}^{\infty} \subset \operatorname{dom}\left(-\Delta_{\alpha_{0}}\right)$. It is easy to check that $\left\|\psi_{n}\right\|_{\mathrm{L}^{2}\left(\Omega_{\epsilon}\right)}=1$, for each $n \in \mathbb{N}$, and $\psi_{n} \stackrel{w}{\longrightarrow} 0$, in $\mathrm{L}^{2}\left(\Omega_{\epsilon}\right)$, and also that $\left(-\Delta_{\alpha}^{\Omega_{\epsilon}}-\lambda\right) \psi_{n} \rightarrow 0$, in $\mathrm{L}^{2}(\Omega)$, since

$$
\left(-\Delta_{\alpha_{0}}^{\Omega_{\epsilon}}-\lambda\right) \psi_{n}=\left[\left(-\Delta^{\mathbb{R}}-t\right) \phi_{n}\right] \phi_{0}+\left[\left(-\Delta_{\alpha_{0}}^{I}-\mu_{0}\right) \phi_{0}\right] \phi_{n} \longrightarrow 0 .
$$

Hence $\left\{\psi_{n}\right\}_{n=1}^{\infty}$ is a singular Weyl sequence for $-\Delta_{\alpha_{0}}^{\Omega_{\epsilon}}$ at $\lambda$ and, by Lemma 4.9, $\lambda \in \sigma_{\text {ess }}\left(-\Delta_{\alpha_{0}}^{\Omega_{\epsilon}}\right)$.

Proposition 4.11 For each $\alpha_{0} \in \mathbb{R}, \sigma_{\mathrm{ess}}\left(-\Delta_{\alpha_{0}}^{\Omega_{\epsilon}}\right)=\left[-\alpha_{0}^{2}, \infty\right)$.

Proof. Indeed, by Lemma 4.10 we have that $\left[-\alpha_{0}^{2}, \infty\right) \subset \sigma_{\mathrm{ess}}\left(-\Delta_{\alpha_{0}}^{\Omega_{\epsilon}}\right)$. On the other hand, we have the lower bound $b_{\alpha_{0}}^{\Omega_{\epsilon}} \geq-\alpha_{0}^{2}$, consequently $\sigma_{\text {ess }}\left(-\Delta_{\alpha_{0}}^{\Omega_{\epsilon}}\right) \subset\left[-\alpha_{0}^{2}, \infty\right)$. Therefore, the following holds

$$
\sigma_{\mathrm{ess}}\left(-\Delta_{\alpha_{0}}^{\Omega_{\epsilon}}\right)=\left[-\alpha_{0}^{2}, \infty\right)
$$


Lemma 4.12 Let $\alpha_{0} \in \mathbb{R}$ and $\varphi \in \mathrm{L}^{2}\left(\partial \Omega_{\epsilon}\right)$. Then, there exists a positive constant $C$, depending on $\epsilon$ and $\left|\alpha_{0}\right|$, such that any solution $\psi \in W^{2,2}\left(\Omega_{\epsilon}\right)$ of the boundary value problem

$$
\left\{\begin{array}{c}
(-\Delta-\lambda) \psi=0 \quad \text { in } \quad \Omega_{\epsilon} \\
-\frac{\partial \psi}{\partial y}(x, 0)-\alpha_{0} \psi(x, 0)=\varphi(x, 0) \\
\frac{\partial \psi}{\partial y}(x, \epsilon)+\alpha_{0} \psi(x, \epsilon)=\varphi(x, \epsilon)
\end{array}\right.
$$

with any $\lambda<0$, satisfies the estimate

$$
\|\psi\|_{1,2} \leq C\|\varphi\|_{\mathrm{L}^{2}\left(\partial \Omega_{\epsilon}\right)}
$$

Proof. Multiplying the first equation of (25) by $\bar{\psi}$ and integrating by parts, one can produce the identity

$$
\int_{\Omega_{\epsilon}}|\nabla \psi|^{2} \mathrm{~d} x \mathrm{~d} y+\alpha_{0} \int_{\partial \Omega_{\epsilon}}|\psi|^{2} \nu_{2} \mathrm{~d} \sigma-\lambda \int_{\Omega_{\epsilon}}|\psi|^{2} \mathrm{~d} x \mathrm{~d} y=\int_{\partial \Omega_{\epsilon}} \varphi \bar{\psi} \nu_{2} \mathrm{~d} \sigma
$$

where $\nu_{2}$ denotes the second component of the outward unit normal vector to $\partial \Omega_{\epsilon}$. Using the Schwarz and Cauchy inequalities, recalling that $\left|\nu_{2}\right|=1$, and the embedding of $H^{1}\left(\Omega_{\epsilon}\right)$ in $\mathrm{L}^{2}\left(\partial \Omega_{\epsilon}\right)$, we have, for $t \in(0,1)$,

$$
\begin{aligned}
\left.\left|\int_{\partial \Omega} \alpha_{0}\right| \psi\right|^{2} \nu_{2} \mathrm{~d} \sigma \mid & =\left.\left|\int_{\Omega} \alpha_{0} \frac{\partial}{\partial y}\right| \psi\right|^{2} \mathrm{~d} x \mathrm{~d} y|\leq 2| \alpha_{0} \mid\|\psi\|_{\mathrm{L}^{2}\left(\Omega_{\epsilon}\right)}\left\|\partial_{2} \psi\right\|_{\mathrm{L}^{2}\left(\Omega_{\epsilon}\right)} \\
& \leq t^{-1}\left|\alpha_{0}\right|^{2}\|\psi\|_{\mathrm{L}^{2}\left(\Omega_{\epsilon}\right)}^{2}+t\|\nabla \psi\|_{\mathrm{L}^{2}\left(\Omega_{\epsilon}\right)}^{2} \leq t^{-1}\left|\alpha_{0}\right|^{2}\|\psi\|_{1,2}^{2}+t\|\nabla \psi\|_{\mathrm{L}^{2}\left(\Omega_{\epsilon}\right)}^{2} \\
\left|\int_{\partial \Omega} \varphi \bar{\psi} \nu_{2} \mathrm{~d} \sigma\right| & \leq 2\|\psi\|_{\mathrm{L}^{2}\left(\partial \Omega_{\epsilon}\right)}\|\varphi\|_{\mathrm{L}^{2}\left(\partial \Omega_{\epsilon}\right)} \leq t^{-1}\|\varphi\|_{\mathrm{L}^{2}\left(\partial \Omega_{\epsilon}\right)}^{2}+t\|\psi\|_{\mathrm{L}^{2}\left(\partial \Omega_{\epsilon}\right)}^{2} \\
& \leq t^{-1}\|\varphi\|_{\mathrm{L}^{2}\left(\partial \Omega_{\epsilon}\right)}^{2}+t \tilde{C}\|\psi\|_{1,2}^{2}
\end{aligned}
$$

where $\tilde{C}$ is the constant from the embedding of $H^{1}\left(\Omega_{\epsilon}\right)$ in $\mathrm{L}^{2}\left(\partial \Omega_{\epsilon}\right)$. By the above estimates, we obtain

$$
\left(1-t-\lambda-t\left|\alpha_{0}\right|^{2}-t \tilde{C}\right)\|\psi\|_{1,2}^{2} \leq t^{-1}\|\varphi\|_{\mathrm{L}^{2}\left(\partial \Omega_{\epsilon}\right)}^{2} .
$$

The desired conclusion follows by choosing $t>0$ small enough so that the coefficient of $\|\psi\|_{1,2}^{2}$ becomes positive.

Proposition 4.13 Suppose that $\alpha \in W^{1, \infty}(\mathbb{R})$. If $\lim _{|x| \rightarrow+\infty}\left(\alpha(x)-\alpha_{0}\right)=0$, then for each $\lambda \in \rho\left(-\Delta_{\alpha}^{\Omega_{\epsilon}}\right) \cap \rho\left(-\Delta_{\alpha_{0}}^{\Omega_{\epsilon}}\right)$ the operator $\left(-\Delta_{\alpha}^{\Omega_{\epsilon}}-\lambda\right)^{-1}-\left(-\Delta_{\alpha_{0}}^{\Omega_{\epsilon}}-\lambda\right)^{-1}$ is compact in $\mathrm{L}^{2}\left(\Omega_{\epsilon}\right)$.

Proof. Due to the first resolvent identity, it is enough to prove the result for a negative $\lambda$ in the intersection of the respective resolvent sets. Consider $\left\{\phi_{j}\right\}_{j=1}^{\infty} \subset \mathrm{L}^{2}\left(\Omega_{\epsilon}\right)$ bounded and let $\psi_{j}=\left(-\Delta_{\alpha}^{\Omega_{\epsilon}}-\lambda\right)^{-1} \phi_{j}-\left(-\Delta_{\alpha_{0}}^{\Omega_{\epsilon}}-\lambda\right)^{-1} \phi_{j}$; note that $\psi_{j}$ satisfies the first equation in (25). Moreover, inserting $\psi_{j}$ into the second or third equation we obtain

$$
\left(\frac{\partial}{\partial_{y}}+\alpha_{0}\right) \psi_{j}=\left(\frac{\partial}{\partial_{y}}+\alpha_{0}\right)\left(\left(-\Delta_{\alpha}^{\Omega_{\epsilon}}-\lambda\right)^{-1} \phi_{j}-\left(-\Delta_{\alpha_{0}}^{\Omega_{\epsilon}}-\lambda\right)^{-1} \phi_{j}\right)=\left(\alpha_{0}-\alpha\right) \operatorname{tr}\left(-\Delta_{\alpha}^{\Omega_{\epsilon}}-\lambda\right)^{-1} \phi_{j}
$$


so that we take now $\varphi=\left(\alpha_{0}-\alpha\right) \operatorname{tr}\left(-\Delta_{\alpha}^{\Omega_{\epsilon}}-\lambda\right)^{-1} \phi_{j}$, where tr denotes the trace operator from $H^{1}\left(\Omega_{\epsilon}\right) \supset \operatorname{dom}\left(-\Delta_{\alpha}^{\Omega_{\epsilon}}\right)$ to $\mathrm{L}^{2}\left(\partial \Omega_{\epsilon}\right)$. By Lemma 4.12, we have

$$
\left\|\psi_{j}-\psi_{k}\right\|_{1,2} \leq C\left\|\left(\left(\alpha_{0}-\alpha\right) \operatorname{tr}\left(-\Delta_{\alpha}^{\Omega_{\epsilon}}-\lambda\right)^{-1}\right)\left(\phi_{j}-\phi_{k}\right)\right\|_{L^{2}\left(\partial \Omega_{\epsilon}\right)} .
$$

Under the assumption that $\beta \operatorname{tr}\left(-\Delta_{\alpha}^{\Omega_{\epsilon}}-\lambda\right)^{-1}$ is a compact operator, it follows that the sequence $\left\{\psi_{j}\right\}_{j=1}^{\infty}$ is precompact in the topology of $H^{1}\left(\Omega_{\epsilon}\right)$, and with a help of the above inequality one can establish that $\left(-\Delta_{\alpha}^{\Omega_{\epsilon}}-\lambda\right)^{-1}-\left(-\Delta_{\alpha_{0}}^{\Omega_{\epsilon}}-\lambda\right)^{-1}$ is a compact operator in $\mathrm{L}^{2}\left(\Omega_{\epsilon}\right)$.

Let us verify the compactness of the operator $\beta \operatorname{tr}\left(-\Delta_{\alpha}^{\Omega_{\epsilon}}-\lambda\right)^{-1}$. One can show that the sequence of operators $\beta_{m} \operatorname{tr}\left(-\Delta_{\alpha}^{\Omega_{\epsilon}}-\lambda\right)^{-1}$ converges to $\beta \operatorname{tr}\left(-\Delta_{\alpha}^{\Omega_{\epsilon}}-\lambda\right)^{-1}$ in norm, since $\| \beta_{m}-$ $\beta \|_{L^{\infty}(\mathbb{R})} \rightarrow 0$ in $\mathrm{L}^{\infty}(\mathbb{R})$. On the other hand, we shall prove that each operator $\beta_{m} \operatorname{tr}\left(-\Delta_{\alpha}^{\Omega_{\epsilon}}-\right.$ $\lambda)^{-1}$ is compact. Indeed, given $\left\{u_{n}\right\}_{n=1}^{\infty}$ bounded in $\mathrm{L}^{2}\left(\Omega_{\epsilon}\right)$ one has $v_{n}=\left(-\Delta_{\alpha}^{\Omega_{\epsilon}}-\lambda\right)^{-1} u_{n}$ bounded in $H^{1}\left(\Omega_{\epsilon}\right)$, then there exists a subsequence, which we still denote by $v_{n}$, and a function $v \in H^{1}\left(\Omega_{\epsilon}\right)$ such that $v_{n} \rightarrow v$ weakly in $H^{1}\left(\Omega_{\epsilon}\right)$. Since $H^{1}\left(\Omega_{\epsilon}\right)$ is compactly embedded in $\mathrm{L}^{2}\left(\Omega_{m}\right)$, where $\Omega_{m}=(-m, m) \times(0, \epsilon) \subset \Omega_{\epsilon}$, due to the Rellich-Kondrachov theorem (see [1], Sec. VI.), then $v_{n} \rightarrow v$ in $\mathrm{L}^{2}\left(\Omega_{m}\right)$. According to the definition of $\beta_{m}$, we have

$$
\left\|\beta_{m} \operatorname{tr}\left(v_{n}\right)-\beta_{m} \operatorname{tr}\left(v_{l}\right)\right\|_{\mathrm{L}^{2}\left(\partial \Omega_{\epsilon}\right)}=\left\|\beta\left(\operatorname{tr}\left(v_{n}\right)-\operatorname{tr}\left(v_{l}\right)\right)\right\|_{\mathrm{L}^{2}\left(\partial \Omega_{m}\right)} \leq C_{m}\|\beta\|_{\infty}\left\|v_{n}-v_{l}\right\|_{\mathrm{L}^{2}\left(\Omega_{m}\right)} .
$$

It follows that $\beta_{m} \operatorname{tr}\left(v_{n}\right)$ is a Cauchy sequence and thus $\lim _{n \rightarrow \infty} \beta_{m} \operatorname{tr}\left(v_{n}\right)$ exists, for each positive integer $m$. Therefore, the operators $\beta_{m} \operatorname{tr}\left(-\Delta_{\alpha}^{\Omega_{\epsilon}}-\lambda\right)^{-1}$ are compact.

Theorem 4.14 Let $\alpha \in W^{1, \infty}(\mathbb{R})$. If $\lim _{|x| \rightarrow+\infty}\left(\alpha(x)-\alpha_{0}\right)=0$, then

$$
\sigma_{\mathrm{ess}}\left(-\Delta_{\alpha}^{\Omega_{\epsilon}}\right)=\left[-\alpha_{0}^{2}, \infty\right)
$$

Proof. According to Proposition 4.13, the operator $\left(-\Delta_{\alpha}^{\Omega_{\epsilon}}-\lambda\right)^{-1}-\left(-\Delta_{\alpha_{0}}^{\Omega_{\epsilon}}-\lambda\right)^{-1}$ is compact in $\mathrm{L}^{2}\left(\Omega_{\epsilon}\right)$; then, by Theorem XIII.14 in [22], the essential spectrum of $-\Delta_{\alpha}^{\Omega_{\epsilon}}$ and $-\Delta_{\alpha_{0}}^{\Omega_{\epsilon}}$ are identical.

\subsection{Existence of discrete spectrum}

Now, based on [15, 16, 19] and under appropriate conditions, we shall give a variational argument to conclude that $\sigma\left(-\Delta_{\alpha}^{\Omega_{\epsilon}}\right) \cap\left(-\infty,-\alpha_{0}^{2}\right) \neq \emptyset$. This, together with Theorem 4.10 , implies that the spectrum below $-\alpha_{0}^{2}$ is nonempty and formed by isolated eigenvalues of finite multiplicity, i.e., $\sigma_{\text {disc }}\left(-\Delta_{\alpha}^{\Omega_{\epsilon}}\right) \neq \emptyset$.

Theorem 4.15 Suppose that $\left(\alpha(x)-\alpha_{0}\right) \in W^{1, \infty}(\mathbb{R})$, with $\alpha_{0}>0\left(\alpha_{0}<0\right)$. If, moreover, $\left(\alpha(x)-\alpha_{0}\right)$ is integrable with

$$
\int_{\mathbb{R}}\left(\alpha(x)-\alpha_{0}\right) \mathrm{d} x>0 \quad\left(\int_{\mathbb{R}}\left(\alpha(x)-\alpha_{0}\right) \mathrm{d} x<0\right)
$$

and $\lim _{|x| \rightarrow+\infty}\left(\alpha(x)-\alpha_{0}\right)=0$, then

$$
\inf \sigma\left(-\Delta_{\alpha}^{\Omega_{\epsilon}}\right)<-\alpha_{0}^{2}
$$


Proof. Following [15], we wish to obtain a trial function $\psi$ from the form domain of $-\Delta_{\alpha}^{\Omega_{\epsilon}}$ such that the quadratic form $Q_{\alpha}^{\Omega_{\epsilon}}(\psi)<0$, where

$$
Q_{\alpha}^{\Omega_{\epsilon}}(\phi)=b_{\alpha}^{\Omega_{\epsilon}}(\phi)+\alpha_{0}^{2}\|\phi\|_{2}^{2}, \quad \operatorname{dom} Q_{\alpha}^{\Omega_{\epsilon}}=\operatorname{dom} b_{\alpha}^{\Omega_{\epsilon}} .
$$

Let $\zeta$ be a cut-off function, that is, we fix a function $\zeta \in \mathrm{C}_{0}^{\infty}(\mathbb{R})$, with $0 \leq \zeta \leq 1$, and $\zeta \equiv 1$ on $(-1 / 4,1 / 4), \zeta \equiv 0$ on $\mathbb{R} \backslash(-1 / 2,1 / 2)$ and $\|\zeta\|_{2}=1$. Given $\phi_{0}$ as defined in the proof of Lemma 4.10. Consider the sequence $\left\{u_{n}\right\}_{n=1}^{\infty}$ of functions into $\operatorname{dom} b_{\alpha}^{\Omega_{\epsilon}}$, defined by $u_{n}(x, y)=f_{n}(x) \phi_{0}(y)$ where $f_{n}(x)=\zeta(x / n)$. By integration by parts and using the boundary conditions of $\phi_{0}$, we obtain

$$
Q_{\alpha}^{\Omega_{\epsilon}}\left(u_{n}\right)=n^{-1}\left\|\zeta^{\prime}\right\|_{2}^{2}+\left\|f_{n}\right\|_{2}^{2} \int_{0}^{\epsilon}\left(\left|\partial_{y} \phi_{0}\right|^{2}+\alpha_{0}^{2}\left|\phi_{0}\right|^{2}\right) \mathrm{d} y+\int_{\mathbb{R}} \alpha(x)\left|f_{n}\right|^{2}\left(\left|\phi_{0}(\epsilon)\right|^{2}-\left|\phi_{0}(0)\right|^{2}\right) \mathrm{d} x .
$$

Since

$$
\int_{0}^{\epsilon}\left(\left|\partial_{y} \phi_{0}\right|^{2}+\alpha_{0}^{2}\left|\phi_{0}\right|^{2}\right) \mathrm{d} y=-\alpha_{0}\left(\left|\phi_{0}(\epsilon)\right|^{2}-\left|\phi_{0}(0)\right|^{2}\right)
$$

we have

$$
Q_{\alpha}^{\Omega_{\epsilon}}\left(u_{n}\right)=n^{-1}\left\|\zeta^{\prime}\right\|_{2}^{2}+\left(\left|\phi_{0}(\epsilon)\right|^{2}-\left|\phi_{0}(0)\right|^{2}\right) \int_{\mathbb{R}}\left(\alpha(x)-\alpha_{0}\right)\left|f_{n}\right|^{2} \mathrm{~d} x,
$$

since $\left|f_{n}(x)\left(\alpha(x)-\alpha_{0}\right)\right| \leq\left|\alpha(x)-\alpha_{0}\right|$ and $f_{n}(x) \rightarrow 1$ as $n \rightarrow \infty$, we can apply the Dominated Convergence Theorem, because $\left|\alpha(x)-\alpha_{0}\right| \in \mathrm{L}^{1}(\mathbb{R})$, to get

$$
\lim _{n \rightarrow \infty} Q_{\alpha}^{\Omega_{\epsilon}}\left(u_{n}\right)=\left(\left|\phi_{0}(\epsilon)\right|^{2}-\left|\phi_{0}(0)\right|^{2}\right) \int_{\mathbb{R}}\left(\alpha(x)-\alpha_{0}\right) \mathrm{d} x<0 .
$$

Then, there exists some $u_{N} \in \operatorname{dom} b_{\alpha}^{\Omega_{\epsilon}}$ such that $b_{\alpha}^{\Omega_{\epsilon}}\left(u_{N}\right)<-\alpha_{0}^{2}$. It follows that, by invoking Rayleigh-Ritz Theorem, inf $\sigma\left(-\Delta_{\alpha}^{\Omega_{\epsilon}}\right)<-\alpha_{0}^{2}$. Consequently, $\sigma\left(-\Delta_{\alpha}^{\Omega_{\epsilon}}\right) \cap\left(-\infty,-\alpha_{0}^{2}\right) \neq \emptyset$.

\section{Acknowledgement}

I thank César Rogégio de Oliveira for valuable discussions. With readiness he spent time answering my questions during our long discussions which have helped to improve this work. This work was supported financially by CAPES (Brazil).

\section{References}

[1] Adams R.A.: Sobolev spaces, Academic Press, New York, 1975.

[2] Borisov D., Krejčiř́k D.: PT-symmetric waveguide, Integral Equations and Operator Theory 62, 489-515.

[3] Chenaud, B., Duclos, P., Freitas, P., and Krejčiřk, D.: Geometrically induced discrete spectrum in curved tubes, Differential Geometry and its Applications 23 (2005) 95105.

[4] de Oliveira, C.R.: Intermediate Spectral Theory and Quantum Dynamics, Birkhuser, Basel (2009).

[5] de Oliveira, C.R., Rossini, A.F.: Effective operators for nonhomogeneous Robin Laplacian in thin two- and three-dimensional curved waveguides. Submitted for publication. 
[6] de Oliveira, C.R., Verri, A.A.: On the spectrum and weakly effective operator for Dirichlet Laplacian in thin deformed tubes, J. Math. Anal. Appl. 381 (2011), 454-468.

[7] Dittrich, J., and Kř́̌ž, J.: Bound states in straight quantum waveguides with combined boundary conditions, Journal of Mathematical Physics 43, 8 (2002), 38923915.

[8] Duclos, P., Exner, P.: Curvature-induced bound states in quantum waveguides in two and three dimensions, Rev. Math. Phys. 7 (1995), 73-102.

[9] Evans L.C.: Partial differential equations, American Mathematical Society, Providence, 1998.

[10] Exner, P., Minakov, A., and Leonid Parnovski, L.: Asymptotic eigenvalue estimates for a Robin problem with a large parameter, Portugal. Math. (N.S.) Portugaliae Mathematica Vol. 71, Fasc. 2, 2014, 1411566 European Mathematical Society DOI 10.4171/PM/1945.

[11] Exner, P., and Šeba, P.: Bound states in curved waveguides, J. Math. Phys. 30 (1989), 25742580 .

[12] Freitas, P., Krejčiřk, D.: Waveguides with combined Dirichlet and Robin boundary conditions, Math. Phys. Anal. Geom. 9 (2006), 335-352.

[13] Friedlander, F., Solomyak, M.: On the spectrum of the Laplacian in a narrow strip, Israel J. Math. 170 (2009), 337-354.

[14] Friedlander, F., Solomyak, M.: On the spectrum of the Laplacian in a narrow infinite strip, Amer. Math. Soc. Transl. 225 (2008), 103-116.

[15] Goldstone J., Jaffe R.L.: Bound states in twisting tubes, Phys. Rev. B, 45 (1992), 1410014107.

[16] Jílek, M.: Straight quantum waveguide with Robin boundary conditions, Symmetry, Integrability and Geometry: Methods and Applications. SIGMA 3 (2007), 108 (12 pages).

[17] Kato, T.: Perturbation theory for linear operators, Springer-Verlag, Berlin, 1966.

[18] Krejčiřík, D., Bla, H. and Znojil, M.: Closed formula the metric in the Hilbert space of a PT-symmetric model., J. Phys. A 39 (2006), 10143-10153.

[19] Krejčiřík, D., Kříž, J.: On the spectrum of curved planar waveguides, Publ. RIMS Kyoto Univ. 41 (2005), 757-791.

[20] Krejčiřk, D. and Zhiqin Lu: Location of the essential spectrum in curved quantum layers, J. Math. Phys. 55, (2014) 083520.

[21] Pankrashkin, K.: On the asymptotics of the principal eigenvalue for a Robin problem wint a large parameter in planar domains, Nanosystems: PhyS, Chemistry, Math, 2013, 4 (4), P. 474483.

[22] Reed M., Simon B.: Methods of modern mathematical physics, V. Analysis of operators, Academic Press, New York, 1978. 